\title{
A Review Study on Vaping and Status of Vaping in India
}

\author{
Vipin Thampi ${ }^{1 *}$, Rajesh Nair ${ }^{1,2}$, Rajan Rawat $^{1}$, Aman Makhaik $^{1}$ and Subhojit Dey ${ }^{3}$
}

${ }^{1}$ Master of Public Health (MPH), Indian Institute of Public Health-Delhi, Sree Chitra Tirunal Institute for Medical Sciences and Technology, Thiruvananthapuram, Kerala, India

${ }^{2}$ Associate Professor, Indian Institute of Public Health-Delhi, Sree Chitra Tirunal Institute for Medical Sciences and Technology, Thiruvananthapuram, Kerala, India ${ }^{3}$ Disha Foundation E-44, Sushant Lok Phase-III, Gurgaon 122003, Haryana, India

*Corresponding author: Vipin Thampi, Master of Public Health (MPH), Indian Institute of Public Health-Delhi, Sree Chitra Tirunal Institute for Medical Sciences and Technology, Thiruvananthapuram, Kerala, India, Tel: +91-8800873972; E-mail: vthampi9@gmail.com

Received date: November 27, 2018; Accepted date: December 14, 2018; Published date: December 21, 2018

Copyright: (c) 2018 Thampi V, et al. This is an open-access article distributed under the terms of the Creative Commons Attribution License, which permits unrestricted use, distribution, and reproduction in any medium, provided the original author and source are credited.

\begin{abstract}
Introduction: Electronic cigarettes (E-Cigarettes) were introduced into the market in 2007 as an alternative method for quitting smoking. Considering the increase in the use of electronic cigarettes, the study aims to perform the comprehensive review of literature on e-cigarettes.
\end{abstract}

Objective: To explore the presence and usage of e-cigarettes in India and to assess whether use of it can be helpful in reduction of behaviour related to smoking cigarettes

Methods: The literature search strategy was developed using the key words based on the objectives and the electronic based data source for identification of papers was done in PubMed and UK electronic research forum was contacted through to extract the articles

Results: There are almost 75 companies which are supplying e-cigarettes through online in India, and there is no brand in India which manufactures its own product except evolve vapors. Limited studies assessing the health effects of e-cigarettes have been performed as most of them are done in the laboratory conditions. The use of ecigarettes was more in current smokers as the usage increasing in adolescents because of the modifiability and similarity with the conventional cigarettes. Randomized control trial (RCT) and cohort studies were not able to generate enough evidence because of the small sample size in the study and lack of previous data available

Conclusion: Well-designed newer generation e-cigarettes trials must be monitored and measured for a longer period so that the safety of the device can be generated.

Keywords: E-cigarettes; Tobacco cessation; Smoking cessation; Nicotine

\section{Implications}

Conventional cigarettes have been used for more than a century and smokers prefer conventional cigarettes as compared to E-cigarettes worldwide. Some studies mention the benefits of E-cigarettes over conventional cigarette and vice-versa. So, it is difficult to conclude that whether vaping reduces morbidity and mortality. The present study has been conducted to understand the status of vaping in India and its efficacy as an alternative product for tobacco cessation.

\section{Introduction}

An electronic cigarette is a handheld electronic device that tries to create the feeling of tobacco smoking it is a form of an ENDS (Electronic Nicotine Delivery System). They are battery-powered devices that delivery nicotine in an aerosol to the user. E-cigarettes contain nicotine cartridges with airflow sensors, but do not burn tobacco [1]. E-cigarette was introduced in the market as an alternative approach/device for quitting smoking [2]. Vaping is the act of inhaling and exhaling the water vapor produced by an electric device called vaporizer [3]. E-cigarettes can be categorized into three groups disposable, rechargeable and modular can be refilled by the user. Ecigarette devices were manufactured mainly in China initially when it was launched into market in 2007 [4]. There are over 466 brands and 7764 unique flavors with about 242 new flavors added per month [5]. E-cigarettes are mostly available online. Dampf, vape stop, greenvapo and evolve are the main online stores available in India [6]. A single disposable E-cigarettes ranges from $\$ 6$ to $\$ 12$ (INR 390-782) and cartridge models are rechargeable and contain pre-filled cartridges starter kits usually range around $\$ 40$ to $\$ 60$ (INR 2600-3900) [7].

The usage of the E-cigarettes is more in France and United Kingdom which represents 6 percent and 3.5 percent of the adult population [1]. In United States e-cigarette usage has been highest among never smokers aged 18-24 y contributing about 9.7 percent of the total population as compared to other age groups [8]. In India, there are almost 75 companies which are supplying e-cigarettes through online in India according to association of vapers India and use is more common in adolescents because consumption of e-cigarettes occurs through online and there is no authority website protection for ecigarette advertisement and it is available in various flavors. States like Karnataka, Kerala, Punjab, J\&K, Mizoram and Maharashtra have banned e-cigarettes under the Drugs and Cosmetics Act, 1940, and 
Page 2 of 4

Food Safety \& Standards (Prohibition and Restriction on Sales) Regulation, 20119.

Conventional cigarettes have been used for more than a century and smokers are preferring conventional cigarettes as compared to ecigarettes worldwide, more information about the long term health hazards of the conventional cigarettes is available, whereas e-cigarettes are newer products which has been implemented 10 y before because of this the data available on the long term health consequences is limited. Some studies are mentioning the benefits of e-cigarettes over conventional cigarette and in contrast other studies are mentioning its health hazards equal to conventional cigarette so it is difficult to come to a conclusion that whether the product reduces morbidity and mortality. The present study has been conducted to understand the status of vaping and its efficacy as an alternative product for tobacco cessation.

\section{Methods}

\section{Data sources and searches}

Literature search strategies was developed using medical subject headings (MESH) based on the terms "electronic cigarette(s)" or "ecig" or "electronic nicotine delivery system (s)" or "alternative-nicotine delivery devices" or "vaping" and "status and "quit" or "smoking cessation" or "smoking reduction". This electronic based search strategy was used for identification of published papers in PubMed. Search results were not limited by language, but all identified studies were in English. This strategy for the date bases was run or conducted from January 2007 to March 2018.

A total of 11 unique articles met the inclusion criteria. Articles titles and abstracts were screened based on the status of vaping and its role on tobacco cessation in addition relevant reports, articles, journals were referred from electronic cigarette research website.

\section{Criteria for considering studies for the review}

Inclusion criteria: Eligible study designs included systematic reviews and meta-analysis, analytical study designs (case-control studies, cohort studies, and analytical cross-sectional studies), descriptive studies (case report, case series, cross-sectional studies); qualitative research and outcome studies on the status and the efficacy of ecigarettes as a smoking cessation or reduction device were included. We considered all study populations that were defined as "adult" by the study authors. The studies that defined adult smokers, current smokers, former smokers, and dual users were used for further analysis.

Exclusion criteria: Editorials, conference papers, studies which were unrelated to topic concerned and studies were excluded. At the end articles relevant will be reviewed.

\section{Data extraction}

A standardized data extraction form was developed to collect information from the selected studies done on e-cigarettes and vaping. Standard data describing each study was extracted, this includes general information of the study (author, year of publication), study characteristics (study design, study location), primary and secondary outcome measures and end points, study strengths and limitations.

\section{Selection of studies}

Studies were reviewed based on the exclusion and inclusion criteria, in three stages.

During the first stage of title screening, titles of the studies identified from the search were assessed for inclusion.

If the study was related to the objectives of the current study, then it was moved to abstract screening. In the next stage of abstract screening, abstracts of these selected titles that were approved and included for the final stage of full text screening.

In the third stage of full text screening, full texts of abstracts selected in the previous stage were screened for eligibility.

\section{Discussion}

Initial searches conducted via PubMed using the key words electronic cigarette, e-cigarette, vaping, and electronic nicotine delivery systems, yielded a total of 537 records were retrieved through the Search Strategy, of which 188 articles were screened for eligibility after full text screening. Finally, 11 articles were included for the review in which one article could be extracted from the first objective (status of vaping in India) and nine articles were extracted related to the second objective (harm reduction approach-smoking reduction) and Figure 1 shows the flow chart of the search strategy.

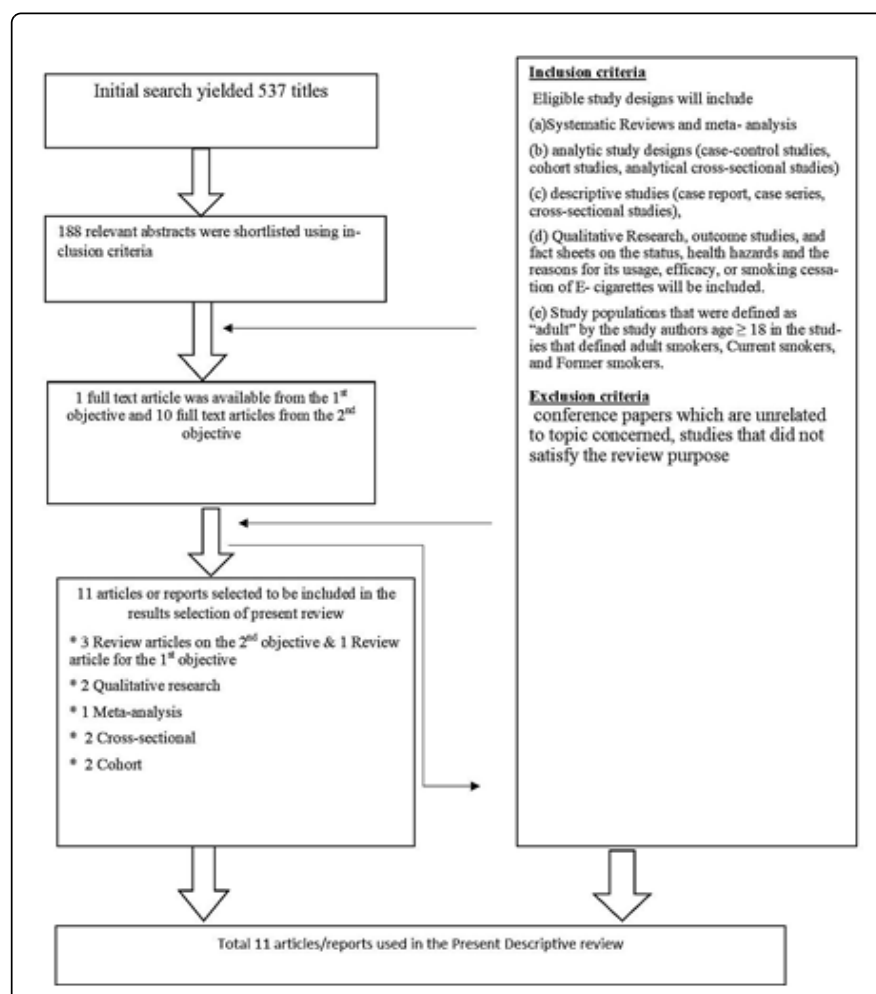

Figure 1: Flow chart of search strategy.

\section{To know the presence and usage of e-cigarettes in India}

The data on E-cigarettes in India both in offline outlets, as well as online retail websites, is still not available. E-cigarettes are available in wide variety of flavors like chocolate, strawberry, blueberry etc. flavors 
Page 3 of 4

similar to cigarette flavors such as tobacco, classic original, cool menthol etc. to more exotic flavors such as cappuccino, double apple, Virginia leaf etc. One can also find dessert flavors such as caramel mocha etc. and the possibilities are truly unimaginable and endless $[9,10]$. Nicotine strength will depend on your current smoking habits or choice of cigarette you smoke. If you are a heavy smoker then $1.8 \%$, $1.6 \%$, or a similar \%, is a good starting point. A lighter smoker could start with $1.2 \%, 0.8 \%$ or similar and someone who prefers no nicotine can choose $0 \%$ [10]. A study conducted in India to check for the online ENDS availability reported that findings out of 65 models (34 brands) which include 45 models of electronic cigarettes, 12 e-shisha, 2 ehookah and 6 e-cigar. In these products information regarding the ingredients, flavors, nicotine strength and claims such as "health related-no tar no harm environmental friendly" were mentioned [11]. But studies on e-cigarettes are claiming it as not an eco-friendly product as it contains substances like formaldehyde, acetaldehyde and acrolein that are released into the environment which are classified as carcinogens by International Agency for Cancer Research (IARC). Apart from that multiple studies have reported both acute and longterm effects on health so long-term studies on humans must be done on this topic in order to generate evidence. Few states in India such as Punjab, Karnataka, and Maharashtra have taken steps to cease the sale of ENDS in areas under their jurisdictions [12].

\section{To assess whether use of e-cigarettes can be helpful in reduction of behaviour related to smoking cigarettes}

E-cigarette manufacturers and users suggest that e-cigarettes are used as smoking-cessation aids as a large proportion of users manage to quit smoking or substantially reduce smoking consumption [13-16], but these studies used convenience samples and suffer from selection bias and do not generalize the general population. In a study conducted on the customers approaching vape shops assessed the smoking status of participants by measuring exhaled carbon monoxide and identified that 66 percent of participants had quit smoking [17]. This study cannot access the random sample of users and could overrepresent the dedicated and advanced consumers. Randomized controlled trials represent the gold-standard in assessing the efficacy of any medical intervention. Studies have shown better results for newer generation and modest results for first generation [18]. Many cohort studies have shown mixed results with some showing the odds of quitting while others showing the opposite effect [19-21]. Metaanalysis has also shown mixed results and systematic review have reported that the E-cigarettes help in quitting smoking [22,23]. Another systematic review found that E-cigarette use was associated with 28 percent reduced chances of quitting [24], while the most recent one found limited evidence for a positive or negative effect of $\mathrm{E}$ cigarettes on smoking cessation, again rating the evidence as low or very low certainty [25]. The systematic reviews were of good quality, there were major problems in the studies that were included to the analyses and were rated 'low 'by GRADE standards due to the small number of trials, low event rates and wide confidence intervals around the estimated means. There is evidence based on the meta-analysis done that usage of E-cigarettes leads to use of conventional cigarettes. There is a reverse temporal association also that usage of combustible tobacco cigarettes at the baseline was positively associated with the use of E-cigarettes. Follow up must be done for 6-12 months' period. The two randomized controlled trials used outdated and poor-quality products that were already withdrawn and replaced by more advanced products at the time of the studies' publication $[26,27]$. The third study provided nicotine-containing E-cigarette liquids for a limited period of time, while for the rest of the study follow up participants could not easily obtain such liquids because they were banned in that country at the time of the study [28]. Cohort studies suffered from very strong bias as many of them included subjects who had already failed to quit smoking with the use of E-cigarettes at baseline, resulting in bias of the outcome being present at the start of the studies [21]. Additionally, they usually assessed ever or current (past $30 \mathrm{~d}$ ) use, definitions that include a lot of experimenters rather than regular users [29]. Population studies have shown that E-cigarettes have helped people quit smoking, with increasing proportions of E-cigarette users being former smokers. There has been an increase in prevalence of $\mathrm{E}$ cigarette use from 700,000 in 2013 to 2.9 million in 2017 [30]. The use of the E-cigarettes has risen from $33 \%$ in 2012 to $52 \%$ in 2017. In the European Union, an estimated 6.1 million smokers have managed to quit smoking with the help of E-cigarettes, while an additional 9.2 million smokers have reduced their smoking consumption [31]. When current and daily e-cigarette use was assessed separately from ever-use reported smoking cessation and reduction rates were by far higher [31,32]. Cross sectional studies showed $60 \%$ higher odds of quitting smoking as compared to other nicotine replacement therapy [33]. Cross-sectional studies also have serious limitations, such as the lack of temporal association and causality, self-report bias and no objective assessment of the smoking status or the duration of smoking cessation. Also, these studies fail to explore how many of those who claim that they have stopped with the aid of e-cigarettes would have stopped anyway and how many of those who used an e-cigarette but failed to stop would have stopped had they used another method [34].

\section{Conclusion}

The present study concluded that there is a lack of data regarding the use of E-cigarettes and its safety and efficacy. In order to cover these lacunae, we need to give emphasis on the reporting of E-cigarette in the GATS (Global Adult Tobacco Survey). To examine whether Ecigarettes can be used as a source of harm reduction in the population and to find out the effects of E-cigarettes on them, extensive research should be carried out to explore the complex interactions between smoking and E-cigarette.

\section{Strength}

This study performs the comprehensive review of literature to know the status and presence of E-cigarettes in India and the efficacy of the E-cigarettes as a smoking cessation device globally.

\section{Conflict of Interest}

There was no conflict of interest.

\section{Funding}

No funding source available.

\section{Acknowledgement}

I would like to thank faculties of my institution Indian Institute of Public Health, Delhi (IIPH) for giving me support and guiding me in completing my research, I would like to thank my supervisor Dr. Rajesh Nair without his support this research would have been not possible. 


\section{References}

1. SEATCA_E-Cig (2014) Electronic cigarettes in Asia a review of promotions and availability. SEATCA_E-Cig.

2. Heydari G, Ahmady AE, Chamyani F, Masjedi M, Fadaizadeh L, et al. (2017) Electronic cigarette, effective or harmful for quitting smoking and respiratory health: A quantitative review papers. Lung India 34: 25.

3. Ernst, Young LLP (2016) E cigarette. EY-E-Cigarette Rep.

4. Grana R, Benowitz N, Glantz SA (2014) E-cigarettes: a scientific review. Circulation 129: 1972-1986.

5. Huey S (2018) Escape the vape: Health hazards of the latest nicotine craze. Nursing 2018.

6. Online Shopping India (2017) Which are the best online stores for buying electronic cigarettes \& liquids in India? Quora.

7. IEC Vaping Authority (2018) E-cigarettes vs. cigarettes: A cost analysisIEC Vaping Authority. IEC Best E-Cig and Vape Guides.

8. Schoenborn CA, Gindi RM (2015) Electronic cigarette use among adults: United States, 2014. NCHS Data Brief 217: 1-8.

9. Sushmi D (2017) Govt likely to stub out 'toxic' e-cigarettes. The Times of India.

10. Litejoy (2018) How to choose your Eliquid-Beginner's Guide. Litejoy.

11. Mohanty VR, Chahar P, Balappanavar AY, Yadav V (2017) Electronic nicotine delivery systems (ENDS): Mapping the Indian online retail market. Nicotine Tob Res 19: 1386-1389.

12. Zee Media Bureau (2016) Karnataka becomes third state to ban ecigarettes in the country. ZEENEWS.

13. Dawkins L, Turner J, Roberts A, Soar K (2013) "Vaping" profiles and preferences: an online survey of electronic cigarette users. Addiction 108: 1115-1125.

14. Farsalinos KE, Romagna G, Tsiapras D, Kyrzopoulos S, Voudris V, et al. (2014) Characteristics, perceived side effects and benefits of electronic cigarette use: A worldwide survey of more than 19,000 consumers. Int J Environ Res Public Health 11: 4356-4373.

15. Farsalinos KE, Romagna G, Tsiapras D, Kyrzopoulos S, Spyrou A, et al. (2013) Impact of flavour variability on electronic cigarette use experience: an internet survey. Int J Environ Res Public Health 10: 7272-7282.

16. Gucht DV, Adriaens K, Baeyens F (2017) Online vape shop customers who use e-cigarettes report abstinence from smoking and improved quality of life, but a substantial minority still have vaping-related health concerns. Int J Environ Res Public Health 7: E798.

17. Tackett AP, Lechner WV, Meier E (2015) Biochemically verified smoking cessation and vaping beliefs among vape store customers. Addiction 110: 868-874.

18. Pasquale C, Davide C, Fabio C, Jaymin BM, Massimo C, et al. (2013) Efficiency and safety of an electronic cigarette (ECLAT) as tobacco cigarettes substitute: a prospective 12-month randomized control design study. Plos One 9.
19. Biener L, Hargraves JL (2015) A longitudinal study of electronic cigarette use among a population-based sample of adult smokers: association with smoking cessation and motivation to quit. Nicotine Tob Res 17: 127-133.

20. Brose LS, Hitchman SC, Brown J, West R, McNeill A, et al. (2015) Is the use of electronic cigarettes while smoking associated with smoking cessation attempts, cessation and reduced cigarette consumption? A survey with a 1-year follow-up. Addiction 7: 1160-1168.

21. Vickerman (2013) Use of electronic cigarettes among state tobacco cessation quitline callers. Oxford Acad 10: 1787-1791.

22. Hartmann-Boyce J, McRobbie H, Bullen C, Begh R, Stead LF, et al. (2016) Electronic cigarettes for smoking cessation. Cochrane Database Syst Rev 9: CD010216.

23. McRobbie H, Bullen C, Hartmann-Boyce J, Hajek P (2014) Electronic cigarettes for smoking cessation and reduction. Cochrane Database Syst Rev. 12: CD010216.

24. Kalkhoran S, Glantz SA (2016) E-cigarettes and smoking cessation in real-world and clinical settings: a systematic review and meta-analysis. Lancet Respir Med 4: 116-128.

25. Dib RE, Suzumura EA, Akl EA (2017) Electronic nicotine delivery systems and/or electronic non-nicotine delivery systems for tobacco smoking cessation or reduction: a systematic review and meta-analysis. BMJ Open 7: e012680.

26. Manzoli L, Flacco ME, Fiore M (2015) Electronic cigarettes efficacy and safety at 12 months: Cohort study. Plos One 10: e0129443.

27. Bullen (2013) Electronic cigarettes for smoking cessation: a randomised controlled trial. The Lancet.

28. Adriaens K, Van Gucht D, Declerck P, Baeyens F (2014) Effectiveness of the electronic cigarette: An eight-week Flemish study with six-month follow-up on smoking reduction, craving and experienced benefits and complaints. Int J Environ Res Public Health 11: 11220-11248.

29. Michael SA, Raymond GB, David L (2016) How to define e-cigarette prevalence? Finding clues in the use frequency distribution. HHS Public Access E1: 24-29.

30. ASH (2017) Use of e-cigarettes among adults in Great Britain 2017 Action on Smoking and Health.

31. Farsalinos KE, Poulas K, Voudris V, Le Houezec J (2016) Electronic cigarette use in the European Union: analysis of a representative sample of 27460 Europeans from 28 countries. Addiction 111: 2032-2040.

32. Farsalinos KE, Poulas K, Voudris V, Le Houezec J (2017) Prevalence and correlates of current daily use of electronic cigarettes in the European Union: analysis of the 2014 Eurobarometer survey. Intern Emerg Med 12: 757-763.

33. Brown J, Beard E, Kotz D, Michie S, West R, et al. (2014) Real-world effectiveness of e-cigarettes when used to aid smoking cessation: a crosssectional population study. Addiction 109: 1531-1540

34. Maziak (2017) Eurobarometer survey and e-cigarettes: unsubstantiated claims. Addiction 112: 545 